\title{
Topographical Maps as Complex Networks
}

\author{
Luciano da Fontoura Costa and Luis Diambra \\ Institute of Physics of São Carlos. University of São Paulo, \\ São Carlos, SP, Caixa Postal 369, 13560-970, phone +55 163373 9858, \\ FAX +55162 33739879 , Brazil, luciano@if.sc.usp.br
}

(Dated: 13 Agosto 2004)

\begin{abstract}
The neuronal networks in the mammals cortex are characterized by the coexistence of hierarchy, modularity, short and long range interactions, spatial correlations, and topographical connections. Particularly interesting, the latter type of organization implies special demands on developing systems in order to achieve precise maps preserving spatial adjacencies, even at the expense of isometry. Although object of intensive biological research, the elucidation of the main anatomic-functional purposes of the ubiquitous topographical connections in the mammals brain remains an ellusive issue. The present work reports on how recent results from complex network formalism can be used to quantify and model the effect of topographical connections between neuronal cells over the connectivity of the network. While the topographical mapping between two cortical modules are achieved by connecting nearest cells from each module, four kinds of network models are adopted for implementing intra modular connections, including random, preferential-attachment, short-range, and long-range networks. It is shown that, though spatially uniform and simple, topographical connections between modules can lead to major changes in the network properties in some specific cases, depending on intra modular connections schemes, fostering more effective intercommunication between the involved neuronal cells and modules. The possible implications of such effects on cortical operation are discussed.
\end{abstract}

PACS numbers: 87.19.La, 45.53.+n, 89.75.k, 89.75.Hc

\section{INTRODUCTION}

Among the vast spectrum of natural phenomena involving intense information exchange between spatially distributed elements, the mammals cortex stands out as particularly complex and intriguing [1]. While the full understanding of this organ still represents one of the biggest challenges to science, continuing investigations in the areas of neuroanatomy, neurophysiology, neurogenetics, and neuroinformatics have provided a wealthy of information about its organizational principles. It is currently known that the mammals neocortex is characterized by coexistence of hierarchy, modularity, short and long range connections, spatial correlations, and topographical maps. Two neuronal modules are said to be topopographically connected if adjacent neurons of the input layer connect to adjacent neurons of the output layer (e.g. [2, 3]). One of the most distinctive and ubiquitous properties of the mammals brain are the topographical connections between its several modules [3, 4, 5]. In the visual system, for example, the cortical region LGN (i.e. lateral geniculate nucleus) connects topographically to the cortical region V1, and then to V2 and further (e.g. 6, 7, 8]). The fact that such modules exchange information vertically along the hierarchies while communicating horizontally with other modules in the same hierarchical level has motivated a computational model known as multistage integration [5]. Indeed, cortical feedback through such connections seems to be essential for achieving important functionalities such as orientation selectivity [9]. Sensory cortical modules are not only topographically connected between themselves, but also receive topographically structured representations of the input stimuli. In the visual system, for instance, we have the visiotopic maps which have been identified as being important for target detection [10]. At the same time, the functional characteristics of the cells distributed along the cortical surface have been shown to be highly correlated, in the sense that cells that are close one another tend to have similar functions (e.g. 3, 11). It is very likely that such organizational principles are not accidental or secondary. Contrariwise, it is possible that such geometrical arrangement of the cortical circuitry may be essential for proper information processing. The main purpose of the present work is to analyze modular topographical connections in terms of the interesting and powerful concepts and measurements supplied by the new area of complex networks. The basic structure of the cortical connections, which is shared by many species, is likely to be the result of an attempt at optimizing several conflicting requirements simultaneously, including minimal wiring, minimal metabolism/energy, number of cortical areas, as well as molecular and genetical constraints 12. The minimal wiring requirement, and henceforth minimal transmission delay, have often been identified in the literature as the most important requirement shaping cortical connections [13, 14, 15, 16]. The special efforts invested by the central nervous system in implementing topographical connections provide a primary indication that such a kind of strategy plays an important role in minimizing connectivity requirements while guaranteeing effective cortical processing.

The importance of maintaining spatial relationships and adjacencies through several cortical modules can, in principle, be associated to the following putative requirements or properties derived from experimental findings 
and computational theory:

Adjacency: As extensively indicated by experimental investigations, neighboring neurons tend to have similar functionalities, implying spatial correlation of neuronal activity along the cortical surface. Such an organization also accounts for a certain degree of redundancy.

Accessibility: Neural operation involves intensive exchange of information along time and space. In order that decisions can be taken timely, it is important that neurons enrolled in cooperative processing have effective access to information in any of the enrolled cells. Information accessibility can be quantified in terms of time or distance, and can be estimated inside the same cortical module or between different modules. High accessibility demands more connections between neurons, with the highest possible degree being achieved when each cell is directly connected to every other cell. In other words, connectivity tends to favor accessibility.

Parallelism: As neuronal cells are relatively slow processing units, real-time cortical operation requires parallel and distributed processing. It is important to observe that some parallel processing paradigms, such as vector processing and pipelining, do not necessarily lead to intense combinatorial connections between all involved modules.

Broadcasting: Another important mechanism possibly underlying information transmission is broadcasting. Unlike point-to-point intercommunication, broadcasting is characterizing by the fact that the same information is sent to several other neurons. Broadcasting can be particularly useful for neural modulation and control. While wide broadcasting can take place along time, short term action demands high levels of neuronal connections.

Though it is not currently clear how these features are adopted and combined at different cortical regions in order to allow emergence of proper behavior, it is only through the quantitative characterization of network connectivity and spatial constraints that new hypotheses and further experimental investigation, including functional evaluation, can be obtained and validated. In order to better appreciate the possible implications of topographical connections for cortical architecture, it is also important to consider the connectivity patterns intrinsic to cortical modules. As there is no current agreement on whether the local cortical connections follow random [17] or selective attachment [18, 19], both situations are considered in the present study.

While the connections underlying traditional neuronal networks can naturally be represented in terms of graphs, the recent interest in complex networks [20] has paved the way to characterize important properties of such structures with respect to both their connections and organization, especially in terms of the node degree (i.e. the number of connections of each neuron), average length and clustering coefficient 20, 21, 22]. Graph theory 21] thus provides several concepts and tools for measuring, modeling and validating several aspects of cortical geometry and functionality.
The present work reports on the potential of applying complex network formalism to investigate in a quantitative manner the effects that topographical connections may have in modifying important properties of the involved cortical modules, with special attention given to their connectivity. The neuronal cells, each represented by a network node, are assumed to be uniformly distributed along the cortical topography. The connections inside each modular network follow four different architectures: preferential-attachment (PAT), random network (RAN), short-range network (SHR) and long-range network (LNR), which will be detailed later. Topographical connections between two such cortical modules $\mathrm{A}$ and $\mathrm{B}$ are established by linking each node of $\mathrm{A}$ to the nearest node in $\mathrm{B}$ with probability $\beta$. In order to quantify the impact of such topographical connections over the network properties, several measurements are obtained while the degree of topographical coupling between modules $\mathrm{A}$ and $\mathrm{B}$, quantified in terms of the probability $\beta$, is increased. Furthermore, we measure the same network properties for random connections between $\mathrm{A}$ and $\mathrm{B}$ in order to establish a comparison with the topographical connections case. We also consider uni- and bidirectional connections between modules for both topographical and random inter-modular models, as further explained below.

The article starts by introducing the adopted network terminology as well as the several measurements considered for characterization of the properties of the investigated networks. The obtained simulation results characterize the changes of the network properties in terms of the topographical coupling, indicating that topographical connections can have major impact over the properties of the involved networks.

\section{MODELS AND METHODS}

The cortical modules, with $N$ nodes connected through $n$ directed edges, are embedded into an $L \times L$ twodimensional space $\Omega$ representing the cortical domain associated to each module. Each network node $i$, $i=1,2, \ldots, N$, is randomly positioned at coordinate $\left(x_{i}, y_{i}\right)$. The number of nodes in each module is $N=$ Round $\left(\gamma L^{2}\right)$ where $\gamma$ is the density of nodes and Round is the rounding function. The connections inside each cortical module are implemented as follows: a pair of selected nodes establishes a connection if a random number, uniformly distributed between $[0,1]$, is smaller than $p$. The selection of nodes to be connected is performed according to the following architectures: PAT, RAN, SHR or LNR. In the PAT networks, the connections follow a preferential attachment scheme as described in the following. The probability to choose a node to implement a connection depends linearly on the number of connections of that node. Our procedure began with the same probability for all nodes and new connections are added progressively. This procedure results in a network with 
(a) PAT
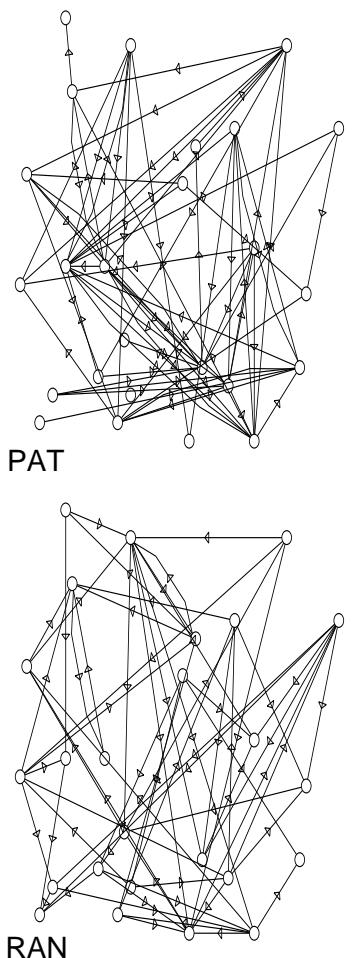

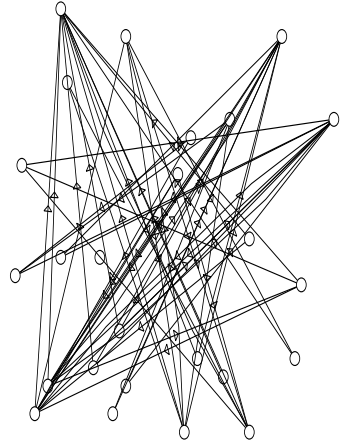

(b) LNR

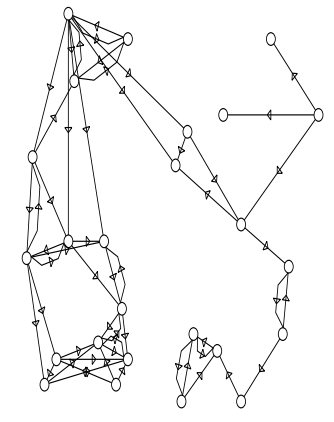

(d) SHR

FIG. 1: Diagram representing the four typical network modules used in this work: preferential-attachment (A), long range (B), random (C) and short range (D). The spatial distribution of nodes is the same for all diagrams. We considered $L=50, p=0.1, \gamma=0.01$ (i.e. $N=25$ nodes), and $T=0.03$ for the cases $\mathrm{B}$ and $\mathrm{D}$.

few highly connected nodes and many poorly connected nodes (Figure 1-a). For RAN networks, two nodes are selected at random for respective connection (Figure 마. c). The construction of the SHR and LNR networks are similar but follow different criteria. First, a list of pair of nodes with Euclidean distances in decreasing or increasing order is elaborated (for SHR or LNR networks, respectively). Then a pair of nodes is randomly selected and if a random number, uniformly distributed between $[0,1]$, is smaller than $\exp [-i /(T N)]$, the pair is connected, where $i$ is the ordinal number of the pair in the list and $T$ is a dimensionless parameter that regulates the length of the connection. Small values of $T$ produce networks that observe the imposed criteria more closely, while for very large values of $T$ a RAN architecture is obtained (see Figures 마 b and 1 1 d). In this paper we set $T=0.1$ in order to retain some longer or shorter range intra-modular connections for SHR and LNR networks, respectively. For all cases the boundary conditions of modules are open (i.e. non-periodical).

Random and topographical schemes were considered for the inter-modular connections (IMC). In the topographical case, the IMC between neurons in the two cortical modules $\mathrm{A}$ and $\mathrm{B}$ are established according to the

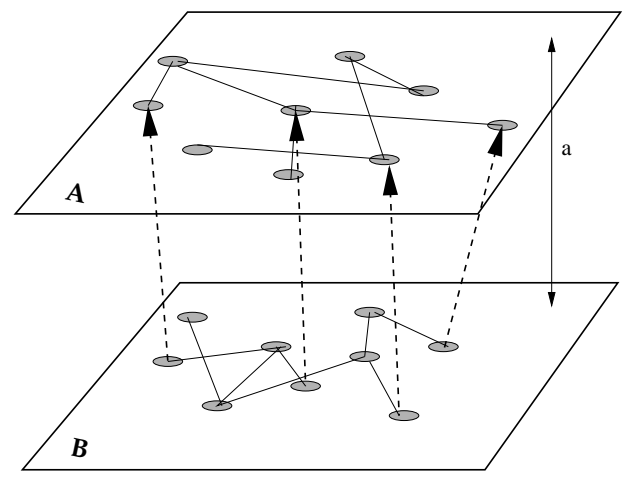

FIG. 2: Diagram representing unidirectional topographical connections (dashed lines) from nodes of module B to nodes of module A, which are separated by distance $a$.

following rule: each neuron in module A connects in a directed way to the nearest neuron in module $\mathrm{B}$ with probability $\beta$. The nearest neighbor condition is ignored in the case of the random model. Therefore, the probability $\beta$ controls the degree of topographical coupling between the two modules, with complete mapping being achieved for $\beta=1$. The two modules $\mathrm{A}$ and $\mathrm{B}$ are adjacent and they are separated by a distance $a$, so that nodes in B do not overlap with nodes in A (see Figures 2] and [5. Both topographical and random IMC models were implementing regarding bidirectional or unidirectional connections. In the bidirectional case, there exist directed connections from nodes of module $\mathrm{A}$ to nodes of module $\mathrm{B}$, and vice-versa from $\mathrm{B}$ to $\mathrm{A}$, while for unidirectional connections, there exist directed connections solely from nodes of module B to nodes in module A.

The following measurements were used in this work in order to quantify several properties of the considered complex networks:

Shortest path length: Let $i$ and $j$ be two network nodes with at least one path from $i$ to $j$. The shortest path, $\delta_{i, j}$, between these nodes is understood as the minimal total sum of edge lengths connecting $i$ to $j$. The average of this measurement, denoted by $\langle\delta\rangle$, can be used to characterize the accessibility between two nodes in terms of Euclidean arc length. When there are no paths from $i$ to $j$, we set $\delta_{i, j}=(N-1) \sqrt{\left(2 L^{2}+a^{2}\right)}$ in order to penalize the accessibility in that case. We calculate the mean over all shortest paths of the network in the bidirectional case, while for unidirectional case the averaging procedure is applied only over $\delta_{i, j}$ such that $i$ and $j$ are nodes from B and A layers respectively, as illustrated in Figure 2

Path degree: Is the fraction of pair of nodes without any path between them. Represented henceforth as $\langle\pi\rangle$, this measurement provides complementary information about the accessibility between nodes. 

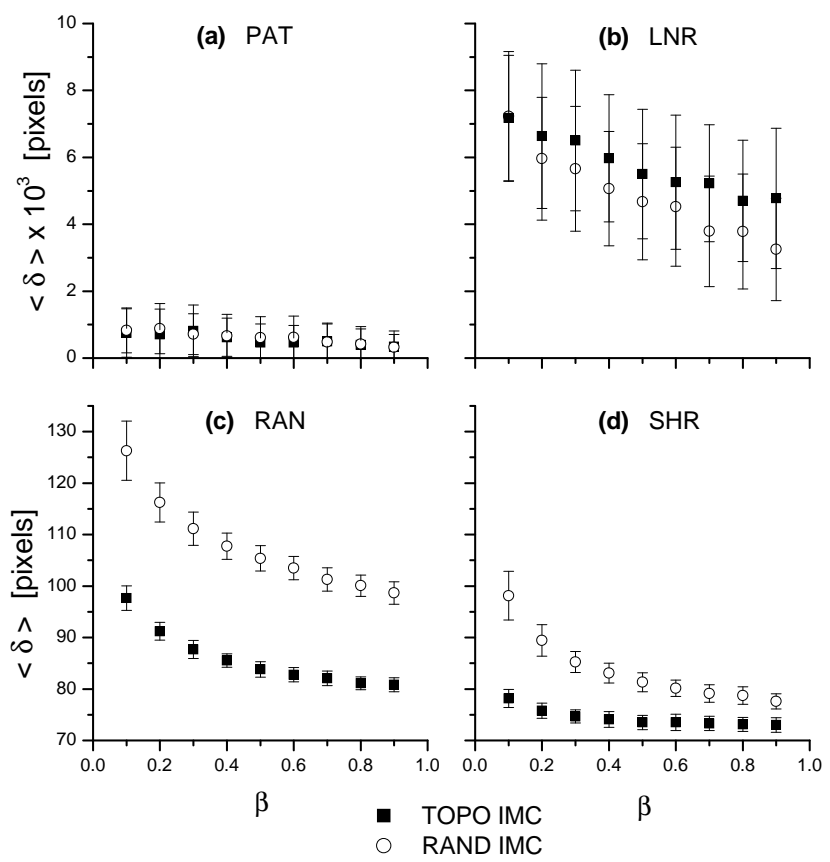

FIG. 3: The average and standard deviation of the shortest path lengths for unidirectional IMC networks with $p=$ 0.1. We consider four different modular architectures: preferential-attachment (a), long range (b), random (c) and short range (d). Filled squares correspond to topographic IMC and open circles to random IMC. The scale of the vertical axes in (b) and (d) panels are the same as in (a) and (c) respectively.

\section{RESULTS}

One hundred realizations of each models were obtained by simulations considering $L=128$ pixels, $\gamma=0.02$, $p=0.1$ and 0.3 . For short range networks, we used $T=0.1$, while the values of $\beta$ vary from 0.1 to 0.9 . The adopted cortical separation was $a=8$ pixels, similar to the typical distance between neighboring nodes inside a module.

Figures 3 and 4 show the average shortest path length $\langle\delta\rangle$ as a function of the unidirectional IMC intensity quantified by $\beta$, for $p=0.1$ and $p=0.3$, respectively. The values $\langle\delta\rangle$ decrease linearly in the PAT and LNR architectures (Figures 3(a-b) and 4(a-b)), while the RAN and SHR architectures present an exponential decay of $\langle\delta\rangle$ when $\beta$ increases (Figures 3 (c-d) and $4(\mathrm{c}-\mathrm{d})$ ). The IMC type, either topographical or random, influences the behavior of $\langle\delta\rangle$ in a selective manner, depending on the connectivity model adopted for the modules. For example, in PAT and LNR architectures the behavior of $\langle\delta\rangle$ for random IMC is essentially undistinguishable from the topographical IMC case. The greatest difference appears for RAN and SHR architectures: in these cases the topographical connections have a lower average shortest path
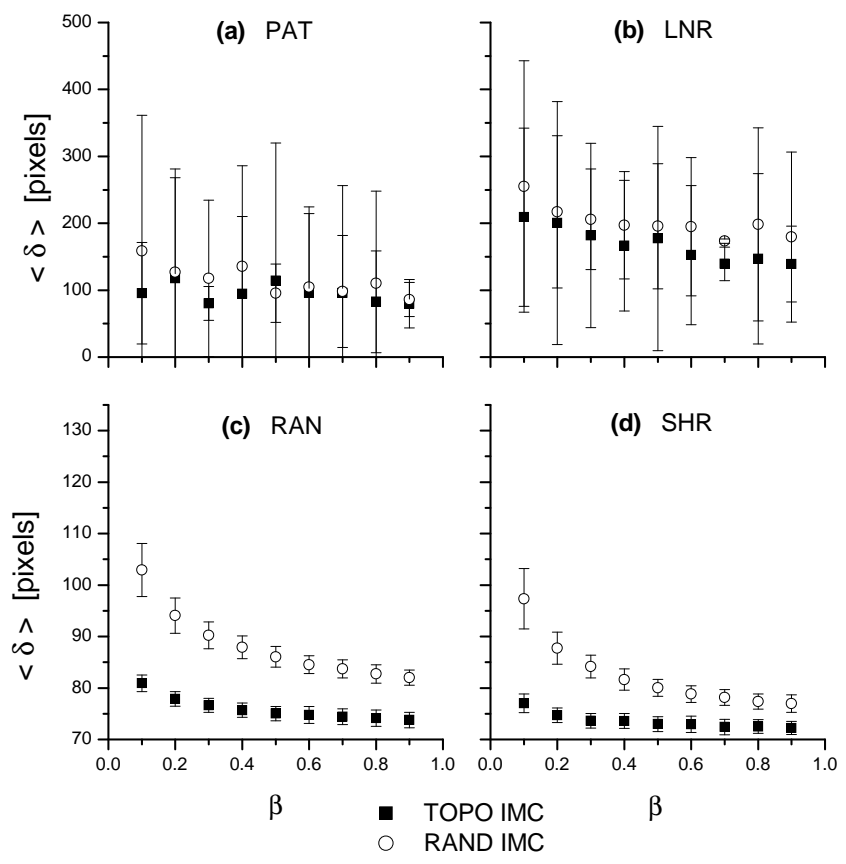

FIG. 4: The average and standard deviation of shortest path lengths for unidirectional IMC networks with $p=0.3$. We consider four different modular architectures: preferentialattachment (a), long range (b), random (c) and short range (d). Fill square corresponds to topographic IMC and open circle to random IMC. The scale of the vertical axes of (b) and (d) panels are the same that (a) and (c) respectively.

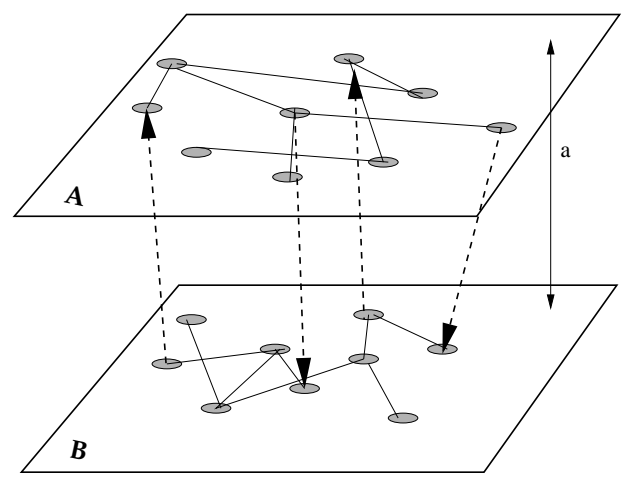

FIG. 5: Diagram representing two network modules (A and B) separated by distance $a$ and connected bidirectionally by topographical connections (dashed lines).

than the corresponding random IMC. The difference between topographical and random IMC is more evident in the RAN architecture for $p=0.1$. In addition, when $p$ increases from 0.1 to $0.3,\langle\delta\rangle$ decreases by about $20 \%$ in the RAN architecture for both topographical and random IMCs, while in the SHR architecture $\langle\delta\rangle$ does not present significant changes. This is in contrast to the strong decrease in $\langle\delta\rangle$ when $p$ increases from 0.1 to 0.3 in the PAT 

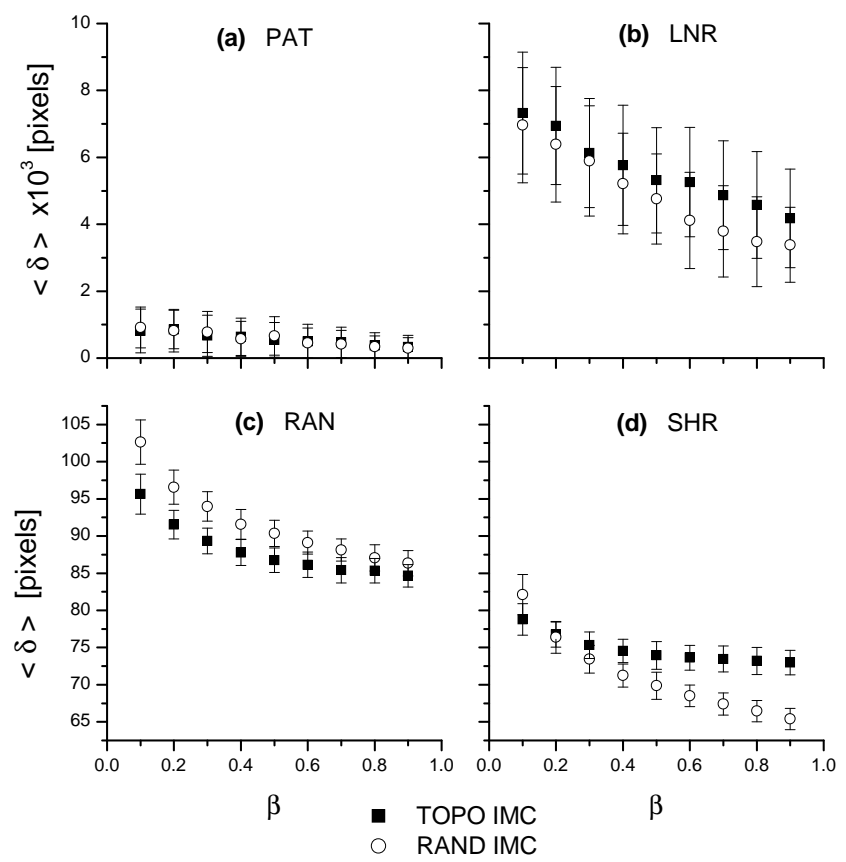

FIG. 6: The average and standard deviation of shortest path lengths for bidirectional IMC networks with $p=0.1$. We consider four different modular architectures: preferentialattachment (a), long range (b), random (c) and short range (d). Fill square corresponds to topographic IMC and open circle to random IMC. The scale of the vertical axes of (b) and (d) panels are the same that (a) and (c) respectively.

and LNR architectures, particularly in the latter case, which always showed $\langle\delta\rangle$ higher than the former.

Figures [6] and 7 display the average shortest path length, $\langle\delta\rangle$ as a function of the IMC intensity $\beta$, for $p=0.1$ and $p=0.3$ respectively, regarding bidirectional connections. We observe that the IMC type, either topographical or random, influences the behavior of $\langle\delta\rangle$ in a selective manner, depending on the modular architecture. In particular, $\langle\delta\rangle$ associated to random IMC is lower than that corresponding to topographical IMC only for the SHR architecture. In the case of the other modular architectures there are no great differences. In a similar way to the unidirectional case, $\langle\delta\rangle$ decreases linearly in the PAT and LNR architectures (Figures [6(a-b) and 7 (ab)), and exponentially in the RAN and SHR architectures (Figures 6(c-d) and 7 (c-d)). In addition, we observe that topographical IMC decays slower than random IMC. The difference between the two types of IMC is more definite in the SHR architecture for $p=0.3$. We also note that an increase of $p$ values from 0.1 to 0.3 in the PAT and LNR architectures, particularly in the latter, tend to enhance accessibility between the network nodes in that models (Figures 6(a-b) and [7(a-b)). This effect is substantially weaker in the RAN architecture (particularly in the topographical IMC), and almost null in the SHR architecture both for topographical and random IMCs.
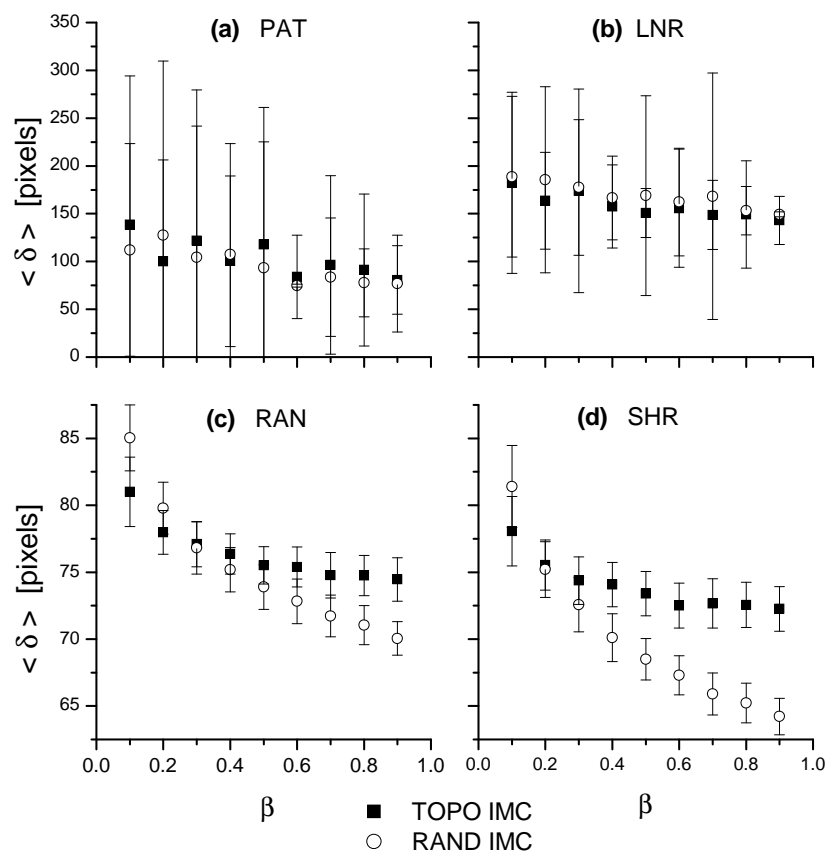

FIG. 7: The average and standard deviation of the shortest path lengths for bidirectional IMC networks with $p=0.3$. We consider four different modular architectures: preferentialattachment (a), long range (b), random (c) and short range (d). Filled squares correspond to topographic IMC and open circles to random IMC. The scale of the vertical axes in (b) and (d) panels are the same as in (a) and (c) respectively.

The properties characterized by the node degree, path degree and clustering coefficient did not show significant differences between topographic and random realizations of the IMC. However, we observed different values of path degree produced by the PAT and LNR when compared to the other two network types. In particular, path degrees tend to be considerably higher in the PAT and LNR than in SHR and RAN networks, which implies a higher standard deviation $\Delta \delta$ (displayed as errors bars in Figures (317). Figure 8 displays the scatter plots of $\langle\pi\rangle$ in terms of the standard deviation $\Delta \delta$ of the shortest path length. We observe that the fraction of pair of nodes without any path connecting them is smaller in the LNR networks than in PAT networks. Moreover $\langle\pi\rangle$ suffers the influence of directionality (uni- or bidirectional) in the PAT networks but not in LNR case. In the latter case, random IMC seems to be more efficient than topographical both for unidirectional and bidirectional cases. The measurements $\langle\pi\rangle$ for RAN and SHR networks are small and almost independent of the IMC type, $p$ and $\beta$ values (for the values considered here), while in PAT networks $\langle\pi\rangle$ decreases with $\beta$ and $p$.

Our simulations suggest that topographical networks are more effective in terms of minimal wiring only for the RAN and SHR architectures with unidirectional IMCs. However, opposite conclusions were reached regarding 


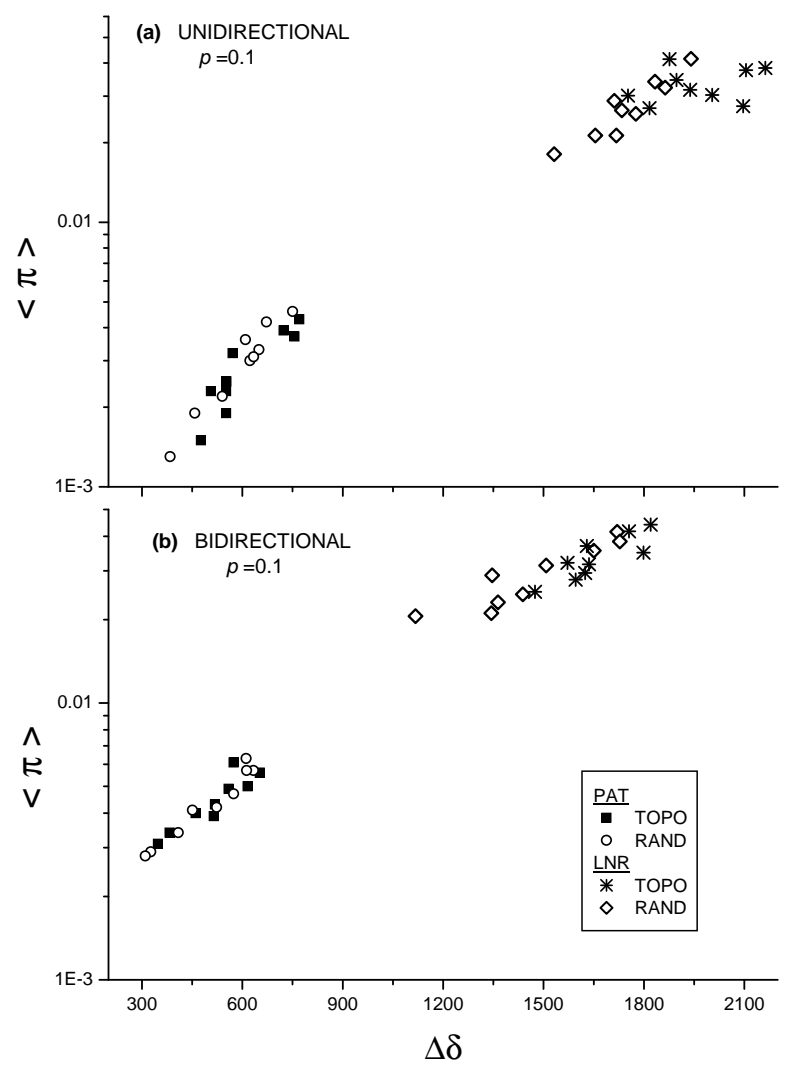

FIG. 8: Fraction of pairs of nodes without a path connecting them, $\langle\pi\rangle$, versus the standard deviation of the shortest path lengths, $\Delta \delta$, for unidirectional (a) and bidirectional (b) connections types. We consider PAT networks with topographical IMC (filled squares), and random IMC (open circles); and LNR networks with topographical IMC (asterisk symbols), and random IMC (open rhomboids).

bidirectional IMCs, except for the RAN architecture for low $p$ and $\beta$. Furthermore, the cortical architectures obtained for the short range networks are, in general, more effective in the sense of minimal wiring than random networks, and less sensitive to the IMC intensity.

\section{CONCLUSIONS}

An interesting conclusion from our experiments is that the PAT and LNR models, irrespective of $p$ or the directionality of the mappings, are little affected by the type of map between modules being random or topographical. In other words, in case the intracortical connections are PAT or LNR, there is little advantage in using topographical maps as the means for getting overall shorter connections. In that case, topographical maps would need to be biologically justified in some other way, perhaps in terms of metabolical constraints. Still regarding the PAT and LNR models, the average shortest path has been found to be always smaller for the former, but such an advantage tends to diminish with $p$. Therefore, preferential-attachment networks such as those considered in this work are particularly efficient for obtaining shortest path connections between two cortical modules irrespective of the type of connection (i.e. random or topographical).

A completely different scenario has been identified for the RAN and SHR models, in the sense that the type of connection between modules tended to influence more definitely the respective average shortest paths. Generally, the SHR tended to have average shortest path $\langle\delta\rangle$ smaller than for RAN networks. Substantial differences of $\langle\delta\rangle$ as consequence of random or topographical connections were observed for SHR model with bidirectional maps and high $p$, with the random connections leading to smaller shortest path values than the topographical case (see Figures-6(d) and-7(d)). However, the greatest differences of $\langle\delta\rangle$ were obtained for the RAN networks with unidirectional maps and for smaller $p$ (see Figures-3 (c) and-4(c)). Interestingly, the topographical connections allowed substantially shortest paths in this case. This effect was also verified, to a lesser degree, for the RAN with higher $p$. From the biological perspective, such results indicate in the case of SHR and RAN cortical modules, the topographical maps lead to substantially smaller values of average shortest paths when one considers unidirectional maps. In this sense, the existence of unidirectional topographical connections in the cortex could be understood as being compatible with random and/or short range intracortical connections, which are the connectivity schemes found to benefit the most from such a kind of mapping.

The main implications for cortical function and organization of the Finding reported in the current work are discussed in the following. First, it is clear that topographical connections, even at moderate levels, can affect the properties of the involved modules. Indeed, the addition of a few short length connections between the two topographically organized modules can considerably enhance the accessibility between any two nodes in the resulting structure, reducing the shortest path between pairs of neurons, with the consequent improvement of time accessibility. Such enhancements imply that information can be exchanged and broadcasted more effectively between the neurons of the resulting topographically connected network than in the cortical modules taken isolated.

The intrinsic properties of topographical connections suggest that the many cortical regions involving such a communication could therefore account for one of the explanations for this ubiquitous and peculiar aspect of cortical architecture, enhancing accessibility while minimizing the length of the IMC, while also preserving spatial adjacency. More general conclusions considering the whole cortex are precluded by the fact that the cortical morphology and mapping seem to vary from species to species and from region to region (e.g. [8] and [25]). At the same time, the use of the concepts and results reported in this paper provides an interesting tool for in- 
vestigating and interpreting each of such cases. Considering that topographical connections coexist with lateral connections at intra-modular level, the above proposed methodology can also be immediately extended to investigate the possible implications of such connections along the cortical modules inside the same module. The consideration of topographical connections between other types of spatially-constrained complex networks can also be considered as a means of enhancing information exchange.

\section{Acknowledgments}

Luciano da F. Costa is grateful to FAPESP (processes 99/12765-2 and 96/05497-3), CNPq and Human Frontier for financial support. Luis Diambra thanks Human Frontier for his post-doc grant.
[1] J. W. Scannell, G. A. P. C. Burns, C. C. Hilgetag, M. A. O' Neil, and M. P. Young, Cereb. Cortex 9, 277 (1999).

[2] D. B. Chklovskii, J. Neurophys. 83, 2113 (2000).

[3] E. R. Kandel, J. H. Schwartz, and T. M. Jessel, Essentials of neural science and behavior (Appleton and Lange, Englewood Cliffs, 1995).

[4] D. V. V. Essen, Nature 385, 313 (1997).

[5] A. Bartels and S. Zeki, Proc. R. Soc. Lond. B 265, 2327 (1998).

[6] Y. Ding and V. A. Casagrande, J. Comp. Neurol. 391, 429 (1998).

[7] J. M. Ichida and V. A. Casagrande, J. Comp. Neurol. 454, 272 (1998).

[8] D. C. Lyon, N. Jain, and J. H. Kaas, J. Comp. Neurol. 401, 109 (1998).

[9] S. G. D. P. C. Murphy and A. M. Sillito, Science 286, 1552 (1999).

[10] M. Kardar and A. Zee, arXiv:physics/02088029 (2002).

[11] K. J. Friston, C. D. Frith, P. Fletcher, P. F. Liddle, and R. S. J. Frackowiak, Cerebral Cortex 6, 156 (1996).

[12] J. Karbowski, J. Comput. Neurosci. 15, 347 (2003).

[13] D. B. Chklovskii T. Schikorski and C. F. Stevens, Neuron 34, 341 (2002).
[14] C. Cherniak, Trends Neurosci. 18, 522 (1995).

[15] J. Karbowski, Phys. Rev. Lett. 86, 3674 (2001).

[16] G. Mitchison, Proc. R. Soc. London Ser. B 245, 151 (1991).

[17] V. Braitenberg and A. Schüz, Cortex: Statistics and Geometry of Neuronal Concectivuty (Springer, Berlin, 1998).

[18] M.P. Young, Nature 358, 152 (1992).

[19] O. Shefi, I. Golding, R. Segev, E. Ben-Jacob, and A. Ayali Phys. Rev. E 66, 21905 (2002).

[20] R. Albert and A. L. Barabási, Rev. Mod. Phys. 74, 47 (2002).

[21] B. Bollobás, Modern Graph Theory (Springer-Verlag, New York, 2002).

[22] A. L. Barabasi, E. Ravasz, and T. Vicsek, Physica A 259, 559 (2001).

[23] S. N. Dorogovtsev and J. F. F. Mendes, Advances in Physics 51, 1079 (2002).

[24] M. E. J. Newman, SIAM Review 45, 167 (2003).

[25] J. H. Kaas and D. C. Lyon, Progr. Brain Res. 134, 285 (2001). 